\title{
Relação entre vulvovaginite pré-natal e laceração perineal relacionada ao parto
}

Relationship between antenatal vulvovaginitis and birth-related perineal tear Relación entre vulvovaginitis prenatal y desgarro perineal relacionado con el parto

\author{
Adriana Amorim Francisco ${ }^{1}$ (i) https://orcid.org/0000-0003-4402-6774 \\ Nayara de Oliveira Alves ${ }^{2}$ iD htps://orcid.org/0000-0001-7519-6923, \\ Mary Steen ${ }^{3}$ ib https://orid.org/0000-0003-2927-6011 \\ Juliana Regina Linfante Andrade ${ }^{1}$ ib https://orid.org/0000-0002-9244-77/4x \\ Marcia Barbieri' ${ }^{1}$ ø nttps://orcid.org/0000-0002-4662-1983 \\ Maria Cristina Gabrielloni1 ${ }^{10}$ hitps://orcid.org/0000-0003-2395-9161
}

Como citar:

Francisco AA, Alves NO, Steen M, Andrade JR, Barbieri M, Gabrielloni MC. Relação entre vulvovaginite pré-natal e laceração perineal relacionada ao parto. Acta Paul Enferm. 2021;34:AAPE002205.

DOI

http://dx.doi.org/10.37689/actaape/2021A0002205

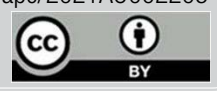

Descritores

Traumatismos do nascimento; Vulvovaginite; Gravidez; Cuidado pré-natal

Keywords

Birth injuries; Vulvovaginitis; Pregnancy; Prenatal care

Descriptores

Traumatismos del nacimiento; Vulvovaginitis: Embarazo; Atención prenatal

\section{Submetido \\ 11 de Agosto de 2020 \\ Aceito \\ 3 de Dezembro de 2020 \\ Autor Correspondente \\ Adriana Amorim Francisco} Email: aamorimfrancisco@yahoo.com.br

\section{Resumo}

Objetivo: Determinar a relação entre vulvovaginite pré-natal e laceração perineal relacionada ao parto.

Método: Estudo transversal analítico com 100 puérperas $\geq 18$ anos de idade que deram à luz por parto normal a um bebê único, vivo, a termo, em apresentação cefálica, em um centro de parto liderado por enfermeiras obstetras. Os dados foram coletados a partir da ficha de pré-natal e nascimento e por entrevista estruturada dos participantes. A distribuição das variáveis contínuas e categóricas de acordo com a ruptura perineal foi comparada com o teste t de Student, qui-quadrado e teste exato de Fisher. Para as variáveis significativamente associadas à ruptura perineal, foi estimado o Odds Ratio com modelos de regressão logística. Modelos de regressão múltipla foram ajustados para avaliar o efeito independente das variáveis. A significância estatística foi considerada com $p<0,05$.

Resultado: A média de idade das participantes foi de 23,1 anos, $16 \%$ dos trabalhos de parto foram induzidos com misoprostol, em 54\% dos trabalhos de parto houve infusão de ocitocina sintética, 83\% dos partos foram em posição de litotomia, 98\% de manobra hands-on, 75\% de laceração perineal, 54\% de vulvovaginite prénatal, média de peso ao nascer, circunferência cefálica e torácica dos recém-nascidos: 3,102g, 33,3cm e $32,2 \mathrm{~cm}$, respectivamente. Vulvovaginite pré-natal $(p=0,005)$, peso ao nascer do recém-nascido $(p=0,006)$ e perímetro cefálico $(0,027)$ tiveram associação com a ruptura perineal. A análise múltipla mostrou que mulheres com vulvovaginite pré-natal tiveram uma chance de 4,6 (IC 95\%: 1,712-14,125; $p=0,004$ ) de sustentar laceração perineal em comparação com aquelas sem vulvovaginite, independentemente do peso do recém-nascido (OR:1,182, IC 95\%: 1,002-1,415; $p=0,056)$ e do perímetro cefálico(OR: 1,160, IC 95\%: $0,721-1892 ; p=0,544)$. Não houve associação entre 0 tratamento de vulvovaginite pré-natal e laceração perineal ( $p>0,999)$ ou vulvovaginite pré-natal e gravidade da laceração perineal (OR:1,061, IC 95\%: 0,383$3,069 ; p=0,911)$.

Conclusão: Este estudo demonstrou associação entre laceração perineal no parto e vulvovaginite pré-natal. É necessário prevenir e tratar a vulvovaginite pré-natal e oferecer cuidados perineais adequados durante o parto às mulheres que tiveram vulvovaginite na gestação.

\section{Abstract}

Objective: To determine the relationship between antenatal vulvovaginitis and birth-related perineal tear.

Methods: An analytical cross-sectional study with 100 postpartum women, $\geq 18$ years of age, who gave birth vaginally to a single, live, full-term baby in cephalic presentation at a midwife-led birth center. Data were collected from the antenatal and birth record and by structured interview of participants. Distribution of continuous and categorical variables according to perineal tear were compared by using the Student's T-test,

'Escola Paulista de Enfermagem, Universidade Federal de São Paulo, São Paulo, SP, Brazil.

${ }^{2}$ Hospital Estadual Vila Alpina, São Paulo, SP, Brazil.

${ }^{3}$ School of Nursing and Midwifery, University of South Australia, Adelaide, Austrália.

Conflitos de interesse: nada a declarar. 
Chi-square and Fisher Exact tests. For variables significantly associated with perineal tear, the Odds Ratio with logistic regression models was estimated. Multiple regression models were adjusted to evaluate the independent effect of variables. Statistical significance was considered at a level $p<0.05$.

Results: mean of participants' age 23.1 years, 16\% labor induced with misoprostol, 54\% synthetic oxytocin infusion in labor, 83\% lithotomy birth position, $98 \%$ "hands on" maneuver, $75 \%$ perineal tear, $54 \%$ antenatal vulvovaginitis, mean of newborn birth weight, head and thoracic circumference: $3.102 \mathrm{~g}$, $33.3 \mathrm{~cm}$ and $32.2 \mathrm{~cm}$, respectively. Antenatal vulvovaginitis $(p=0.005)$ and newborn birth weight $(p=0.006)$ and head circumference $(0,027)$ were associated with perineal tear. The multiple analysis showed that women who had antenatal vulvovaginitis had a 4.6 (IC 95\%:1.712-14.125; $p=0.004)$ chance of sustaining perineal tear compared to those without vulvovaginitis, regardless of newborn birth weight (OR:1.182 IC 95\%:1.002-1.415; $p=0,056)$ and head circumference (OR:1.160 IC 95\%: 0.721-1892; $p=0.544$ ). There was no association between treating antenatal vulvovaginitis and perineal tear ( $p>0,999)$ or antenatal vulvovaginitis and perineal tear severity (OR: 1.061 IC 95\%: 0.383-3.069; $p=0.911$ ).

Conclusion: This study demonstrates an associated risk between antenatal vulvovaginitis perineal injury. It is necessary to prevent and treat antenatal vulvovaginitis, and offer proper perineal care to women who have had antenatal vulvovaginitis during childbirth.

\section{Resumen}

Objetivo: Determinar la relación entre vulvovaginitis prenatal y desgarro perineal relacionado con el parto.

Método: Estudio transversal analítico con 100 puérperas de $\geq 18$ años de edad que dieron a luz por parto vaginal a un bebé único, vivo, a término, en presentación cefálica, en un centro de parto liderado por enfermeras obstetras. Los datos se recopilaron a partir de la ficha de atención prenatal y nacimiento y mediante encuesta estructurada de las participantes. La distribución de las variables continuas y categóricas de acuerdo con la ruptura perineal fue comparada con el test-T de Student, la prueba $\chi^{2}$ de Pearson y la prueba exacta de Fisher. Para las variables significativamente asociadas a la ruptura perineal, se estimó el Odds Ratio con modelos de regresión logística. Se adaptaron los modelos de regresión múltiple para evaluar el efecto independiente de las variables. La significación estadística fue considerada con $p<0,05$.

Resultado: El promedio de edad de las participantes fue de 23,1 años, el $16 \%$ de los trabajos de parto fueron inducidos con misoprostol, en el $54 \%$ de los trabajos de parto hubo infusión de oxitocina sintética, el $83 \%$ de los partos fueron en posición de litotomía, el 98 \% de maniobra hands-on, el 75 \% de desgarro perineal, el $54 \%$ de vulvovaginitis prenatal, el promedio de peso al nacer de 3,102 g, de circunferencia cefálica de 33,3 cm y de circunferencia torácica de $32,2 \mathrm{~cm}$ de los recién nacidos. La vulvovaginitis prenatal $(p=0,005)$, el peso al nacer del recién nacido $(p=0,006)$ y el perímetro cefálico $(0,027)$ tuvieron relación con la ruptura perineal. El análisis múltiple demostró que mujeres con vulvovaginitis prenatal tuvieron una probabilidad de 4,6 (IC 95 \%: 1,712-14,125; $p=0,004$ ) de tener desgarro perineal en comparación con aquellas sin vulvovaginitis, independientemente del peso del recién nacido (OR:1,182, IC 95 \%: 1,002-1,415; p=0,056) y del perímetro cefálico (OR:1,160, IC 95 \%: 0,721-1892; p=0,544). No se observó relación entre el tratamiento de vulvovaginitis prenatal y desgarro perineal $(p>0,999)$ o entre la vulvovaginitis prenatal y la gravedad del desgarro perineal (0R:1,061, IC $95 \%$ : 0,383-3,069; $p=0,911)$.

Conclusión: Este estudio demostró que existe relación entre desgarro perineal en el parto y vulvovaginitis prenatal. Es necesario prevenir y tratar la vulvovaginitis prenatal y ofrecer cuidados perineales adecuados durante el parto a las mujeres que tuvieron vulvovaginitis en el embarazo.

\section{Introdução}

O trauma perineal relacionado ao parto comumente afeta mulheres que dão à luz por parto normal. É caracterizado por laceraçóes, episiotomia ou ambas e está associado a um risco aumentado de lesóes do esfíncter anal, complicaçôes na cicatrização, ${ }^{(1)}$ infecção, distúrbios do assoalho pélvico, ${ }^{(2)}$ dispareunia e dor perineal, ${ }^{(3,4)}$ que impactam adversamente na saúde e no bem-estar pós-parto. ${ }^{(5)}$

Vários fatores de risco para lesão perineal são descritos na literatura e incluem paridade, posição de nascimento, episiotomia, peso do recém-nascido ao nascer, etc. ${ }^{(6,7)} \mathrm{O}$ trauma perineal relacionado ao nascimento também parece ser muito comum entre mulheres com vulvovaginite pré-natal não tratada de forma eficaz durante a gravidez, apesar da ausência de evidências robustas sobre qualquer associação.

Apenas uma pesquisa envolvendo 662 mulheres grávidas, idade gestacional >37 semanas, idade en- tre 15 e 45 anos, que deram à luz por via vaginal, teve como objetivo investigar uma associação entre vaginose bacteriana e trauma perineal relacionado ao parto. Os pesquisadores relataram que a vaginose bacteriana foi testada usando o teste de gradiente de Nugent, que é padrão ouro para o diagnóstico de vaginose bacteriana. A prevalência de rotura perineal de $1^{\circ}$ a $4^{\circ}$ grau foi de $35,8 \%$ e náo foi encontrada nenhuma associação entre essas duas variáveis. ${ }^{(8)}$

Durante a gravidez, o corpo da máe passa por vários ajustes anatômicos e fisiológicos em sua resposta imunológica, sistema endócrino, $\mathrm{pH}$ vaginal e cervical e microbiota vaginal, levando à infecçôes. Portanto, durante a gravidez, as mulheres estão mais suscetíveis à vulvovaginite, que é um processo inflamatório ou infecção do trato genital inferior com vários graus de sinais e sintomas, como prurido, dispareunia, queimação e odor vulvar, alteraçóes na cor e volume da secreção vaginal, hiperemia e edema vulvar. Muitas mulheres grávidas podem ser assintomáticas. ${ }^{(9)}$ 
Candidíase, tricomoníase e vaginose bacteriana são as condiçóes de vulvovaginite mais comuns. As duas infecçóes são causadas por microrganismos específicos: Candida albicans e Trichomonas vaginalis, respectivamente; enquanto a vaginose bacteriana é uma condição clínica caracterizada por uma mudança na microbiota vaginal em direção a espécies bacterianas mais diversas, como Gardnerella vaginallis. ${ }^{(10,11)}$ Existem evidências apoiando uma associação entre vulvovaginite durante a gravidez e aborto espontâneo, parto prematuro, ruptura prematura de membranas, corioamnionite, restrição de crescimento intrauterino, infecçóes congênitas e maior morbimortalidade neonatal. Portanto, essas infecçóes devem ser detectadas precocemente e tratadas durante o período pré-natal. ${ }^{(11)}$ Se a vulvovaginite não for tratada ou tratada inadequadamente, o tecido perineal e a mucosa podem sofrer lesóes à medida que o bebê passa pelo canal de parto. ${ }^{(8)}$

Desta forma, justifica-se investigar a existência de associação entre vulvovaginite pré-natal e trauma perineal relacionado ao parto a fim de melhorar a assistência pré-natal e ao parto.

Este é o objetivo do estudo: investigar a relação entre vulvovaginite e laceração perineal relacionada ao parto.

\section{Métodos}

Estudo transversal analítico, conduzido com todas as mulheres $\geq 18$ anos de idade, sem parto vaginal anterior, que deram à luz por via vaginal a um bebê único vivo a termo em apresentação cefálica, em um centro de parto humanizado liderado por enfermeiras obstetras, de agosto a novembro de 2018. Tratase de um serviço de referência para gestantes de alto risco que vivem na regiáo sudeste da cidade de Sáo Paulo (Brasil) e contabiliza entre 200 e 300 partos/ mês. Possui cinco salas de parto, onde gestantes de baixo a alto risco são atendidas por enfermeiras residentes e enfermeiras obstétricas durante o trabalho de parto, parto e pós-parto imediato. Obstetras e médicos residentes são responsáveis pela internação e cesariana das pacientes. A rotina de atendimento durante o trabalho de parto, parto e pós-parto ime- diato inclui: escolhas compartilhadas de tomada de decisão, dieta leve, água a vontade, deambulação, uso de bola de parto, partograma e infusáo intravenosa de ocitocina sintética para indução do trabalho de parto. Em janeiro de 2018, ocorreram 213 partos normais neste local: $85(39,9 \%)$ mulheres sem parto vaginal anterior, $42(49,4 \%)$ mulheres com traumatismo perineal, sendo $33(78,6 \%)$ e nove $(21,4 \%)$ mulheres com laceração de $1^{\mathrm{a}}$ e $2^{\mathrm{o}}$ grau, respectivamente. Náo houve nenhuma laceração perineal de $3^{\circ}$ ou $4^{\circ}$ grau.

\section{Coleta de dados}

Os dados foram coletados diariamente, de segunda a sexta-feira, por uma aluna de enfermagem obstétrica treinada, extraídos dos prontuários de pré-natal e nascimento e de entrevistas estruturadas com as participantes. Inicialmente, todas as participantes elegíveis foram identificadas por meio do livro de registro de nascimento e após assinarem o termo de consentimento livre e esclarecido, foram entrevistadas e seu cartão de acompanhamento pré-natal verificado.

\section{Variáveis}

- Variável independente: vulvovaginite durante a gravidez confirmada por meio do registro no cartão de acompanhamento pré-natal e durante entrevista estruturada com puérperas.

- Variável dependente: laceração perineal classificada em $1^{\circ}, 2^{\circ}, 3^{\circ}$ ou $4^{\circ}$ grau a partir de documentação em prontuário.

- Outras variáveis: idade materna, uso de misoprostol e ocitocina no trabalho de parto el ou segundo período, posição de parto materno, manobras perineais (técnicas hands-on e hands-off), lubrificação perineal com vaselina no segundo período, peso ao nascer do bebê e circunferência torácica e craniana, tratamento de vulvovaginite pré-natal.

\section{Análise de dados}

A distribuição normal das variáveis contínuas foi testada usando o teste de Shapiro Wilk. Testes paramétricos e correspondentes não paramétricos foram usados para variáveis contínuas com e sem distribuição normal, respectivamente. As variáveis 
contínuas são descritas por meio de médias e desvios-padrão e as variáveis categóricas por meio de frequências absolutas e relativas. Para comparar a distribuição das variáveis contínuas e categóricas de acordo com a laceração perineal relacionada ao parto, foram usados os testes t de Student, qui-quadrado e exato de Fisher. Para variáveis significativamente associadas à laceração perineal relacionada ao parto $(\mathrm{p}<0,05)$, foi estimado o Odds Ratio com modelos de regressão logística. Modelos de regressão múltipla foram ajustados para avaliar o efeito independente de variáveis significativamente associadas à laceração perineal relacionada ao parto nas análises univariadas $(\mathrm{p}<0,005)$. A significância estatística foi considerada para valor de $\mathrm{p}<5 \%$.

\section{Ética}

Atendendo à resolução do Conselho Nacional de Saúde no 466/2012, este estudo foi aprovado pelo Comitê de Ética em Pesquisa (ID: 2.697.543). Todas as mães participaram voluntariamente após assinarem o termo de consentimento livre e esclarecido.

\section{Resultados}

Entre 575 mulheres que deram à luz por parto normal no período de coleta de dados, 190 (33\%) atenderam aos critérios de inclusão, mas 100 (52\%) mulheres foram incluídas, pois $90(48 \%)$ deram à luz durante os fins de semana e tiveram alta para casa na/antes da manhá de segunda-feira.

A média de idade das participantes foi de 23,1 $(\mathrm{DP}=4,8)$ anos. Setenta e cinco por cento das mães tiveram laceraçóes perineais relacionadas ao parto, $53(70,7 \%)$ de $1^{\circ}$ grau e $22(29,3 \%)$ de $2^{\circ}$ grau. Foi registrado que $62,7 \%$ das participantes tiveram vulvovaginite pré-natal, das quais 24 (51\%) foram tratadas durante o pré-natal. Apesar das evidências sugerindo significativa associação entre vulvovaginite pré-natal e laceração perineal relacionada ao parto, não houve associação entre o tratamento de vulvovaginite durante o atendimento pré-natal e laceraçóes perineais relacionadas ao parto.

Dezesseis por cento das participantes tiveram indução do trabalho de parto com misoprostol, 47
(47\%) receberam infusão de ocitocina sintética durante o trabalho de parto e 11 (20,4\%) apenas no segundo período. Não foi encontrada associação entre o uso de misoprostol ou ocitocina durante o trabalho de parto ou segundo período e laceração perineal relacionado ao parto (Tabela 1 ).

A maior parte das participantes neste estudo deu à luz na posição de litotomia, enquanto um pequeno número optou por uma posição vertical, lateral ou quatro apoios. Os dados sobre manobra perineal e lubrificação com vaselina foram obtidos nos registros de apenas 50 participantes, pois essas intervençôes raramente são registradas. Destas, quase todas as parteiras utilizaram a manobra hands on e evitavam aplicar vaselina na região perineal. Não houve associaçáo significativa entre essas variáveis e laceraçóes perineais relacionadas ao nascimento (Tabela 1).

Tabela 1. Variáveis pré-natais e de nascimento entre mulheres com e sem lacerações perineais relacionadas ao parto

\begin{tabular}{|c|c|c|c|}
\hline & \multicolumn{2}{|c|}{ Laceração Perineal } & \multirow[b]{2}{*}{$p$-value } \\
\hline & $\begin{array}{c}\text { Não }(n=25) \\
n(\%)\end{array}$ & $\begin{array}{c}\operatorname{Sim}(n=75) \\
n(\%)\end{array}$ & \\
\hline \multicolumn{4}{|l|}{ Vulvovaginite } \\
\hline Não & $18(72)$ & $28(37,3)$ & 0,005 \\
\hline Sim & $7(28)$ & $47(62,7)$ & \\
\hline \multicolumn{4}{|c|}{ Tratamento pré-natal $(n=54)$} \\
\hline Não & $4(57,1)$ & $23(48,9)$ & $>0,999$ \\
\hline $\operatorname{Sim}$ & $3(42,8)$ & $24(51,1)$ & \\
\hline \multicolumn{4}{|l|}{ Misoprostol } \\
\hline Não & 22(88) & $62(82,7)$ & 0,754 \\
\hline Sim & $3(12)$ & $13(17,3)$ & \\
\hline \multicolumn{4}{|c|}{ Ocitocina no trabalho de parto } \\
\hline Não & $13(52)$ & $40(53,3)$ & $>0,999$ \\
\hline $\operatorname{Sim}$ & $12(48)$ & $35(46,7)$ & \\
\hline \multicolumn{4}{|c|}{ Ocitocina no Segundo Periodo } \\
\hline Não & $13(52)$ & $29(38,7)$ & 0,349 \\
\hline Sim & $12(48)$ & $46(61,3)$ & \\
\hline \multicolumn{4}{|c|}{ Posição de parto } \\
\hline Litotomia & 18(72) & $65(86,7)$ & 0,119 \\
\hline Agachada & $5(20)$ & $4(5,3)$ & \\
\hline Em pé & $0(0)$ & $2(2,7)$ & \\
\hline Outras & $2(8)$ & $4(5,3)$ & \\
\hline \multicolumn{4}{|c|}{ Manobra perineal $(n=49)$} \\
\hline Não & $0(0)$ & $0(0)$ & $>0,999$ \\
\hline $\operatorname{Sim}$ & $6(100)$ & $43(100)$ & \\
\hline \multicolumn{4}{|c|}{ Vaselina no segundo período $(n=50)$} \\
\hline Não & $2(28,6)$ & $29(67,4)$ & 0,089 \\
\hline Sim & $5(71,4)$ & $14(32,6)$ & \\
\hline
\end{tabular}

O peso ao nascer do recém-nascido, a circunferência cefálica e torácica foram em média $3.102 \mathrm{~g}$ ( $\mathrm{DP}=387), 33,3 \mathrm{~cm}(\mathrm{DP}=1,7)$ e 32,2 cm $(\mathrm{DP}=1,2)$, 
respectivamente. Mulheres com laceração perineal relacionado ao parto deram à luz bebês significativamente mais pesados e com perímetro cefálico maior do que aquelas sem laceração. (Tabela 2).

Tabela 2. Comparação do peso do bebê ao nascer, circunferência da cabeça e torácica entre mulheres com ou sem lacerações perineais relacionadas ao parto

\begin{tabular}{|c|c|c|c|c|}
\hline \multirow{2}{*}{ Laceração Perineal } & $\begin{array}{c}\text { Não } \\
(\mathrm{n}=25)\end{array}$ & $\underset{(n=75)}{\operatorname{Sim}}$ & Total & \multirow{2}{*}{$p$-value } \\
\hline & $\begin{array}{l}\text { Média } \\
\text { (DP) }\end{array}$ & $\begin{array}{l}\text { Média } \\
\text { (DP) }\end{array}$ & $\begin{array}{l}\text { Média } \\
\text { (DP) }\end{array}$ & \\
\hline Peso ao Nascer (g) & $\begin{array}{l}2921,20 \\
(408,98)\end{array}$ & $\begin{array}{l}3162,80 \\
(362,57)\end{array}$ & $\begin{array}{l}3102,40 \\
(387,13)\end{array}$ & 0,006 \\
\hline Perímetro Cefálico (cm) & $\begin{array}{l}32,78 \\
(1,41)\end{array}$ & $\begin{array}{l}33,42 \\
(1,17)\end{array}$ & $\begin{array}{l}33,26 \\
(1,26)\end{array}$ & 0,027 \\
\hline Circunferência torácica (cm) & $\begin{array}{l}31,90 \\
(1,93)\end{array}$ & $\begin{array}{l}32,35 \\
(1,63)\end{array}$ & $\begin{array}{l}32,24 \\
(1,71)\end{array}$ & 0,253 \\
\hline
\end{tabular}

A análise de modelo múltiplo mostra que as mulheres com vulvovaginite pré-natal tiveram 4,6 vezes mais chance de ter laceraçóes perineais em comparação com aquelas sem vulvovaginite, independentemente do peso ao nascer e do perímetro cefálico do bebê (Tabela 3).

Tabela 3. Análise de modelos múltiplos para lacerações perineais relacionadas ao parto

\begin{tabular}{l|c|c}
\hline & Odds ratio [IC 95\%] & p-value \\
\hline Intercepto Y (constante) & 1 (Referencia) & \\
\hline Vulvovaginite & $4,674[1,712 ; 14,125]$ & 0,004 \\
Peso ao nascer & $1,182[1,002 ; 1,415]$ & 0,056 \\
Perímetro cefálico & $1,160[0,721 ; 1,892]$ & 0,544 \\
\hline
\end{tabular}

Não houve associação significativa entre vulvovaginite pré-natal (OR:1,061 IC 95\%: 0,3833,$069 ; \mathrm{p}=0,911$ ), peso ao nascer (OR:1,045 IC 95\%: 0,909-1,203; $\mathrm{p}=0,537)$ e perímetro cefálico (OR:1,143 IC 95\%: 0,743-1,791; $\mathrm{p}=0,546)$ do bebê e a gravidade das laceraçóes perineais relacionadas ao parto.

\section{Discussão}

Este estudo explorou uma associação entre vulvovaginite pré-natal e lacerações perineais relacionadas ao parto, visto que foi identificada uma lacuna na literatura sobre esse tópico. Os resultados mostraram que a vulvovaginite pré-natal, o peso ao nascer e o perímetro cefálico do bebê estão associados a lesóes perineais. Mulheres com vulvovaginite pré-natal tive- ram quase cinco vezes mais chance de ter laceraçóes perineais relacionadas ao parto quando comparadas àquelas sem registro de vulvovaginite. No entanto, esse achado deve ser interpretado com cautela devido ao amplo intervalo de confiança. Apesar de não ter encontrado nenhuma associação com a gravidade do trauma perineal, a presença de vulvovaginite pré-natal aumentou a chance de laceração perineal, independentemente das características do recém-nascido.

$\mathrm{Na}$ literatura, as infecçóes vaginais durante o período pré-natal têm demonstrado uma relação com resultados negativos em recém-nascidos, como prematuridade e baixo peso ao nascer. Curiosamente, um exame de Papanicolau de 1344 gestantes brasileiras entre 2005 e 2008 mostrou que 38,24\% estavam infectadas por Gardnerella vaginalis, 33,75\% por Candida spp, 5,92\% por Trichomonas vaginalis e $21,54 \%$ por flora mista (cocos e outros bacilos)..$^{12)}$ Fortes evidências demonstraram que o rastreamento para detecção precoce e tratamento de vulvovaginite (candidíase, tricomoníase e vaginose bacteriana) por volta das 20 semanas de gestação reduz a incidência de bebês prematuros e com baixo peso ao nascer. ${ }^{(13)}$

Mais de $60 \%$ das participantes deste estudo tiveram registro de vulvovaginite pré-natal, mas nem todas foram tratadas antes do parto. A taxa de laceração perineal de $1^{\circ}$ e $2^{\circ}$ grau relacionada ao nascimento foi de $75 \%$. O presente estudo mostrou que ter vulvovaginite pré-natal aumenta a chance de laceraçóes perineais relacionadas ao parto em mulheres grávidas. No entanto, não foi encontrada associação entre mulheres que receberam ou não tratamento pré-natal para vulvovaginite. Por outro lado, uma pesquisa realizada na França envolvendo 662 mulheres grávidas com 37 semanas de gestação e diagnóstico de vaginose bacteriana, não encontrou associação entre esta condição e lacerações perineais relacionadas ao parto. Não está claro se essas mulheres foram tratadas ou não no período pré-natal, pois isso não foi relatado, mas as taxas de trauma perineal com laceraçóes de $1^{\circ}$ a $4^{\circ}$ grau foram de $35,8 \%{ }^{(8)}$, o que é consistente com as taxas de trauma relatadas anteriormente. ${ }^{(14,15)}$

No presente estudo, não foi possível identificar especificamente o microrganismo que gerou a infecção para cada mulher, pois essa informação não esta- 
va disponível no registro de pré-natal, e além disso, a diretriz para tratamento de vulvovaginite pré-natal no município de São Paulo/Brasil é baseada em algoritmos sindrômicos para mulheres com sinais e sintomas de corrimento vaginal. ${ }^{(16)}$ Um diagnóstico mais assertivo ou etiológico pode ser definido com exames laboratoriais sofisticados, o que muitas vezes é problemático, já que esses exames demandam recursos, aumentam o custo do tratamento, exigem que as mulheres façam consultas extras à clínica pré-natal e podem resultar em atrasos no tratamento. Embora este estudo forneça evidências da associação entre vulvovaginite pré-natal e lacerações perineais relacionadas ao parto, os microrganismos específicos não foram identificados.

Este é o primeiro estudo observacional a demonstrar uma associação entre vulvovaginite pré-natal e roturas perineais relacionadas ao parto em uma população com risco de vulvovaginite.

Uma limitação deste estudo foi que as mulheres que deram à luz no fim de semana não foram incluídas por falta de recursos e financiamento limitado. No entanto, as mulheres que deram à luz nos dias de semana foram recrutadas e os dados indicaram uma associação entre vulvovaginisite pré-natal e risco de laceraçóes perineais relacionadas ao parto. Outra limitação desta pesquisa foi encontrar outros estudos relacionados ao tema para comparar os resultados. A falta de informaçóes detalhadas sobre o tratamento da vulvovaginite no prontuário pré-natal também foi identificada como uma limitação. Este estudo foi um piloto e, portanto, só pode fornecer dados e evidências limitados.

\section{Conclusão}

Este estudo demonstrou um risco associado entre vulvovaginite pré-natal, peso ao nascer, perímetro cefálico e lesão perineal. Mais pesquisas são necessárias para confirmar ou refutar essas associaçóes. Mulheres com vulvovaginite pré-natal tiveram 4,6 vezes mais chance de ter laceraçóes perineais relacionadas ao parto em comparação com aquelas sem vulvovaginite pré-natal, independentemente do peso ao nascer e perímetro cefálico do bebê. Devido ao amplo intervalo de confiança, esse achado deve ser interpretado com cautela. Dependendo da extensão do dano, as laceraçóes perineais relacionadas ao parto podem ter um impacto negativo na sexualidade e saúde reprodutiva das mulheres. Portanto, é importante considerar o risco da associação entre vulvovaginite pré-natal e trauma perineal relacionado ao parto. É de vital importância prevenir e tratar a vulvovaginite pré-natal e melhorar as abordagens pré-natais para detectar essa condição. Estudos adicionais são necessários para compreender mais profundamente a relação entre laceração perineal relacionada ao parto e vulvovaginite pré-natal.

\section{Colaborações}

Francisco AA, Alves NO, Steen M, Andrade JRL, Barbieri $M$ e Gabrielloni MC contribuíram com a concepção do projeto, redação do artigo, revisão crítica relevante do conteúdo intelectual e aprovação final da versão a ser publicada.

\section{Referências}

1. Jiang H, Qian X, Carroli G, Garner P. Selective versus routine use of episiotomy for vaginal birth. Cochrane Database Syst Rev. 2017 Feb;2(2):CD000081.

2. Jones K, Webb S, Manresa M, Hodgetts-Morton V, Morris RK. The incidence of wound infection and dehiscence following childbirthrelated perineal trauma: A systematic review of the evidence. Eur $\mathrm{J}$ Obstet Gynecol Reprod Biol. 2019;240:1-8.

3. Steen M, Diaz M. Perineal trauma: a women's health and wellbeing issue. Br J Midwifery. 2018;26(9):574-84.

4. Gommesen D, Nøhr E, Qvist N, Rasch V. Obstetric perineal tears, sexual function and dyspareunia among primiparous women 12 months postpartum: a prospective cohort study. BMJ Open. 2019;9(12):e032368.

5. Manresa M, Pereda A, Bataller E, Terre-Rull C, Ismail KM, Webb SS. Incidence of perineal pain and dyspareunia following spontaneous vaginal birth: a systematic review and meta-analysis. Int Urogynecol J Pelvic Floor Dysfunct. 2019;30(6):853-68.

6. Zhang M, Wang M, Zhao X, Ren J, Xiang J, Luo B, et al. Risk factors for episiotomy during vaginal childbirth: A retrospective cohort study in Western China. J Evid Based Med. 2018;11(4):233-41.

7. Abedzadeh-Kalahroudi M, Talebian A, Sadat Z, Mesdaghinia E. Perineal trauma: incidence and its risk factors. J Obstet Gynaecol. 2019;39(2):206-11.

8. Letouzey V, Bastide S, Ulrich D, Beccera L, Lomma M, de Tayrac R, et al. Impact of bacterial vaginosis on perineal tears during delivery: a prospective cohort study. PLoS One. 2015;10(11):e0139334. 
9. Gondo DC, Duarte MT, Silva MG, Parada CM. Alteração de flora vaginal em gestantes de baixo risco, atendidas em serviço público de saúde: prevalência e associação à sintomatologia e achados do exame ginecológico. Rev Lat Am Enfermagem. 2010;18(5):919-27.

10. Campbell LM, Ferreira RC, Jordão EV. Vulvovaginitis. In: Primo WQ, Corrêa FJ, Brasileiro JP, editors. Manual de Ginecologia da Sociedade de Ginecologia e Obstetrícia de Brasília. Brasília (DF): Luan Comunicação; 2017. p. 369-86.

11. Souza GN, Vieira TC, Campos AA, Leite AP, Souza E. Treatment of vulvovaginitis in pregnancy. Femina. 2012;40:125-8.

12. Bonfanti G, Gonçalves TL. Prevalence of Gardnerella vaginalis, Candida spp. and Trichomonas vaginalis in cytopathological exams of pregnant women attended at the University Hospital of Santa Maria - RS. Rev Saúde Sta Maria. 2010;36(1):37-46.

13. Sangkomkamhang US, Lumbiganon P, Prasertcharoensuk W, Laopaiboon M. Antenatal lower genital tract infection screening and treatment programs for preventing preterm delivery. Cochrane Database Syst Rev. 2015 Feb;1(2):CD006178.
14. Royal College of Obstetricians \& Gynaecologists (RCOG). The Management of Third-and Fourth-Degree Perineal Tears. Green-top Guideline. N.29. London: RCOG; 2015. [cited 2020 Nov 23]. Available from: https://www.rcog.org.uk/globalassets/documents/guidelines/ gtg-29.pdf

15. Australian Commission on Safety and Quality in Health Care (ACSQHC). Third-and fourth-degree perineal tears. In: The second Australian Atlas of Healthcare Variation. Women's health and maternity; Austrália: ACSQHC; 2017. p.221-35. [cited 2020 Nov 23]. Available from: https://www.safetyandquality.gov.au/sites/ default/files/migrated/3.5-Third-and-fourth-degree-perinealtears.pdf

16. São Paulo (cidade). Secretaria Municipal da Saúde. Coordenação da Atenção Básica/Estratégia Saúde da Família. Manual Técnico: saúde da mulher nas Unidades Básicas de Saúde. $4^{a}$ ed. São Paulo: Secretaria Municipal da Saúde; 2016. 96 p. (Série Enfermagem). [citado 2020 Nov 23]. Disponível em: https://www. prefeitura.sp.gov.br/cidade/secretarias/upload/saude/arquivos/ ManualSaudedaMulherv302012017.pdf 